\title{
Trends and Predictors of Oral Anticoagulation in Patients with Atrial Fibrillation: A Serial Cross-Sectional Study from 2011 to 2016
}

\author{
Maria de Fátima de Araújo Geraldes, ${ }^{1,2}$ Eduardo Sahade Darze, ${ }^{1,2}$ Paulo Novis Rocha ${ }^{1}$ \\ Universidade Federal da Bahia, ${ }^{1}$ Salvador, BA-Brazil \\ Hospital Cárdio Pulmonar, ${ }^{2}$ Salvador, BA-Brazil
}

\section{Abstract}

Background: Despite the efficacy of vitamin K antagonists against stroke in patients with atrial fibrillation (AF), the underuse of this therapy is well documented.

Objectives: To evaluate trends and predictors of oral anticoagulants utilization in patients with AF.

Methods: Observational, retrospective, serial cross-sectional study between 2011-2016. Comparisons between groups were performed using the Student t, Mann-Whitney and Chi-square tests. Logistic regression was used to identify independent predictors of anticoagulation. A p value $<0.05$ was considered significant.

Results: A total of 377 patients were analyzed. The mean age was $70 \pm 15$ years; $52 \%$ were male and $75 \%$ were anticoagulated (20\% with VKA and 55\% with DOAC). Over 5 years, the overall frequency of anticoagulation increased by $22.4 \%$. The use of DOACs increased from $29 \%$ to $70 \%$, whereas the use of VKA decreased from $36 \%$ to $17 \%$. The use of antiplatelet agents alone also fell from $21 \%$ to $6 \%$. The predictors of anticoagulation were previous episodes of AF (OR 3.1, p < 0.001), hypertension (OR 3.0, p < 0.001) and HASBLED score (OR 0.5, p < 0.001). The predictors of DOAC use were serum creatinine (OR 0.2, $\mathrm{p}=0.002)$, left atrial size (OR 0.9, $\mathrm{p}=0.003$ ) and biological valve prosthesis (OR 0.1, $\mathrm{p}=0.007)$. Of the 208 patients using DOACs, $63(30 \%)$ received inadequate prescriptions: 5 with severe drug interactions and 58 with incorrect dosing.

Conclusions: Between 2011 and 2016, DOACs were rapidly incorporated into clinical practice, replacing AVKs and antiplatelets, and contributing to greater use of anticoagulation in patients with AF. (Int J Cardiovasc Sci. 2020;33(1):68-78)

Keywords: Atrial Fibrillation; Anticoagulants; Brain Ischemia; Vitamin K/antagonists \& inhibitors; Thromboembolism/ prevention \& control.

\section{Introduction}

Atrial fibrillation (AF) affects about $1-2 \%$ of the world's population and is associated with a five-fold increased risk of stroke. The use of vitamin $\mathrm{K}$ antagonists (VKA) as anticoagulants reduces the risk of stroke or systemic embolism by $64 \%$ and all-cause death by $26 \% \cdot{ }^{1-7}$ However, observational studies have shown that only $50-60 \%$ of patients with AF eligible for the use of anticoagulants are anticoagulated. Reasons for underutilization of VKAs include numerous drug and food interactions and the inconvenience of laboratory monitoring process, which make it difficult to maintain the International Normalized Ratio (INR) in the narrow therapeutic range, placing patients at risk for ischemic and hemorrhagic events. ${ }^{8-10}$

Over the past 10 years, several randomized trials, involving more than 70,000 patients with AF, compared the use of AVK with direct-acting oral anticoagulants (DOACs). In these studies, DOACs were at least as effective as VKAs in preventing thromboembolic events and promoted a significant reduction in the frequency of intracranial hemorrhage. ${ }^{11}$ In addition, DOACs 
do not require laboratory monitoring to determine effective therapeutic level and have few significant drug interactions.

The purpose of this study is to determine the pattern and predictors of the use of anticoagulants in eligible patients with $\mathrm{AF}$ and how fast DOACs are incorporated in this context.

\section{Methods}

\section{Reference population}

Data were collected from an electronic medical record of all hospitalized patients diagnosed with $\mathrm{AF}$ and/or atrial flutter at a private tertiary institution in Salvador, Bahia, from May 23, 2011 (date of approval of dabigatran in Brazil) to June 30, 2016. Patients were screened from ICDs I48 and R00, and only those with documented AF or atrial flutter were recruited.

\section{Study design}

Retrospective observational study based on electronic chart review, with annual cross-sections for five consecutive years (trends study).

\section{Inclusion and exclusion criteria}

Inclusion criteria were: age $\geq 18$ years, AF diagnosis and/or atrial flutter confirmed by ECG and/or Holter. Patients without electronic prescription of discharge were excluded, as this was the source of the data regarding the use of anticoagulant. On readmissions, the most recent admission was chosen for analysis.

\section{Data collected and definitions}

Demographic and anthropometric data were collected, in addition to the cardiovascular risk factors traditionally related to AF (systemic arterial hypertension [SAH], diabetes, valve disease, myocardial infarction, heart failure, history of bleeding, medications). The risk scores for stroke and bleeding were $\mathrm{CHA}_{2} \mathrm{DS}_{2} \mathrm{VASc}$ and HASBLED, validated in international studies. ${ }^{12-14} \mathrm{AF}$ was classified according to the II Brazilian Guidelines for Atrial Fibrillation of the Brazilian Society of Cardiology.$^{15}$ When there was insufficient information for classification, $\mathrm{AF}$ was considered of indefinite duration. Prior AF was defined as the identification of episodes of this arrhythmia prior to the reference admission during review of medical records.
Echocardiographic data were collected from the most recent test, respecting the period of up to 1 year before admission. Valvular heart disease was defined as the presence of any moderate or severe mitral or aortic lesion. The presence of a valve prosthesis was defined by echocardiogram or clinical history.

Electronic prescription of discharge was used to collect information on anticoagulants, antiplatelets and their doses, and other drugs with potential for drug interaction.

All information regarding hemorrhagic events was collected, but only major bleeding - intracranial hemorrhage, need for blood products or corrective surgical treatment - were considered for analysis.

The following were considered absolute contraindications for oral anticoagulants: active bleeding, severe hemorrhagic diathesis, thrombocytopenia $<50,000$, invasive surgery or procedure to be done, major trauma, hemorrhagic stroke, intracranial or spinal tumor, spinal anesthesia, uncontrolled SAH. Relative contraindications included: end-stage neoplasia, active peptic ulcer, advanced dementia (without a caregiver), alcoholism and frequent falls (more than three per year). Patients with zero $\mathrm{CHA}_{2} \mathrm{DS}_{2}$-VASc were not considered candidates for anticoagulation.

Inappropriate use of $\mathrm{DOAC}^{16}$ was defined as follows: prescriptions containing drugs with strong drug interaction potential; dose inconsistent with the dosage recommended by the manufacturer, considering patient's age, weight and renal function; presence of absolute contraindications (mechanical cardiac valve prosthesis or moderate to severe mitral stenosis).

\section{Statistical analysis}

Continuous variables were summarized by mean and standard deviation or median and interquartile distance, as indicated by the frequency distribution. Comparisons of quantitative variables between 2 groups were done using Student's $t$ test for independent samples and Mann-Whitney. Categorical variables were summarized using simple and relative frequencies, compared between groups using the chi-square test. For analysis of correlation between the HASBLED and $\mathrm{CHA}_{2} \mathrm{DS}_{2}$-VASc scores, the Spearman's technique was used.

To identify predictors of anticoagulation and anticoagulant type, we used binary logistic regression. Firstly, we made a selection of variables using univariate logistic regression. The variables associated with anticoagulation and use of DOAC with $\mathrm{p}$ value $<0.05$ in 
the univariate analyses were later included in multivariate logistic regression models using the backward stepwise method. The variables that reached $p<0.05$ in the final analyses were considered statistically significant.

All analyses were conducted in the Statistical Package for the Social Sciences (SPSS) version 23.

\section{Ethical issues of research}

This study was approved in a plenary session at the Ethics Research Committee Prof. Celso Figuerôa, Hospital Santa Izabel on 11-24-2014, according to Resolutions 466/12 and 251/97, protocol number 917.116.

\section{Results}

From 2011 to 2016, there were 464 admissions with diagnosis of AF discharge. Of these, 87 patients were excluded: 28 for not presenting AF (coding error); 26 hospital readmissions; 20 deaths during admission; 13 for not having electronic prescription of discharge. The final population analyzed consisted of 377 patients.

\section{Demographic and clinical data}

Patients were separated into two groups: patients with and without anticoagulants. The group on anticoagulants was subsequently divided into patients using VKA or DOAC (Table 1). The mean age of the patients was $70 \pm 15$ years, and $52 \%$ were male with mean body mass index (BMI) of $27 \pm 6$. Regarding the type of $\mathrm{AF}, 42 \%$ had paroxysmal $\mathrm{AF}, 12 \%$ had persistent $\mathrm{AF}$, $27 \%$ had permanent $\mathrm{AF}, 19 \%$ with indefinite duration; $10 \%$ of the patients had atrial flutter. There was a high prevalence of comorbidities, with $71 \%$ of hypertensive patients, $23 \%$ of diabetics, $26 \%$ with a history of heart failure, $7.4 \%$ with a history of myocardial infarction and $18 \%$ had ischemic stroke. Of the study population, $25 \%$ had moderate or severe valvulopathy, $3.9 \%$ had biological valve prosthesis and $1.8 \%$ had mechanical valve prosthesis (Table 1 ).

Mean $\mathrm{CHA}_{2} \mathrm{DS}_{2}-\mathrm{VASc}$ risk score was $3.4 \pm 2.0$ and the HASBLED score was $1.2 \pm 1.0$, with $11.2 \%$ of these having HASBLED 3 and $79.8 \%$ having $\mathrm{CHA}_{2} \mathrm{DS}_{2}-\mathrm{VASc}$ $\geq 2$ (Chart 1).

\section{Use of anticoagulant therapy}

Considering the total population over a 5-year period, $75 \%$ of the patients were discharged on anticoagulants (20\% received VKA and 55\% received DOACs), 15\% were on antiplatelets alone and $10 \%$ were not on antithrombotic therapy.

\begin{tabular}{|c|c|c|c|c|c|c|}
\hline Variable & $\begin{array}{c}\text { Without } \\
\text { anticoagulant } \\
(n=93)\end{array}$ & $\begin{array}{c}\text { With } \\
\text { anticoagulant } \\
(n=284)\end{array}$ & $\mathrm{p}^{*}$ & $\begin{array}{c}\text { VKA } \\
(n=76)\end{array}$ & $\begin{array}{l}\text { DOACs } \\
(n=208)\end{array}$ & $\mathrm{p}+$ \\
\hline Age (years) & $73 \pm 18$ & $69 \pm 13$ & 0.059 & $70 \pm 13$ & $69 \pm 14$ & 0.493 \\
\hline Male & $42(45.2)$ & $155(54.6)$ & 0.115 & $41(53.9)$ & $114(54.8)$ & 0.897 \\
\hline Weight $(\mathrm{kg}), \mathrm{n}=370$ & $71.8 \pm 15.5$ & $78.4 \pm 20.6$ & 0.002 & $75.8 \pm 21.4$ & $79.3 \pm 20.3$ & 0.207 \\
\hline Height (meters), $\mathrm{n}=370$ & $1.64 \pm 0.11$ & $1.66 \pm 0.10$ & 0.036 & $1.65 \pm 0.10$ & $1.67 \pm 0.10$ & 0.114 \\
\hline SAH & $59(63.4)$ & $210(73.4)$ & 0.052 & $59(77.6)$ & $151(72.6)$ & 0.392 \\
\hline $\mathrm{DM}$ & $27(29)$ & $61(21.5)$ & 0.135 & $18(23.7)$ & $43(20.7)$ & 0.584 \\
\hline $\mathrm{CHF}$ & $23(24.7)$ & $77(72)$ & 0.652 & $30(39.5)$ & $47(22.6)$ & 0.005 \\
\hline $\mathrm{AMI}$ in the past year & $10(10.8)$ & $18(6.3)$ & 0.159 & $5(6.6)$ & $13(6.3)$ & 0.920 \\
\hline TCA in the past year & $4(4.3)$ & $4(1.4)$ & 0.093 & $1(1.3)$ & $3(1.4)$ & 0.936 \\
\hline History of TIA/ischemic stroke & $25(26.9)$ & $42(14.8)$ & 0.008 & $10(13.2)$ & $32(15.4)$ & 0.640 \\
\hline History of hemorrhagic stroke & $0(0)$ & $2(0.7)$ & 0.417 & $1(1.3)$ & $1(0.5)$ & 0.456 \\
\hline History of AF n=357 & $42(50)$ & $191(70)$ & 0.001 & $49(70)$ & $142(69.9)$ & 0.994 \\
\hline
\end{tabular}




\begin{tabular}{|c|c|c|c|c|c|c|}
\hline Variable & $\begin{array}{c}\text { Without } \\
\text { anticoagulant } \\
(n=93)\end{array}$ & $\begin{array}{c}\text { With } \\
\text { anticoagulant } \\
(\mathrm{n}=284)\end{array}$ & $\mathbf{p}^{*}$ & $\begin{array}{c}\text { VKA } \\
(n=76)\end{array}$ & $\begin{array}{l}\text { DOACs } \\
(n=208)\end{array}$ & pt \\
\hline \multicolumn{7}{|l|}{ AF classification $n=339$} \\
\hline Paroxysmal & $35(41.7)$ & $106(41.6)$ & \multirow[b]{3}{*}{0.185} & $20(27.8)$ & $86(47)$ & \multirow{4}{*}{0.002} \\
\hline Persistent & $5(6)$ & 35 (13.7) & & $13(18.1)$ & $22(12)$ & \\
\hline Permanent & $28(33.3)$ & $64(25.1)$ & & $28(38.9)$ & $36(19.7)$ & \\
\hline Undetermined & $16(19)$ & $50(19.6)$ & & $11(15.3)$ & $39(21.3)$ & \\
\hline $\mathrm{CHA}_{2} \mathrm{DS}_{2}-\mathrm{VASc}$ & $4(0$ to 8$)$ & $3(0$ to 9$)$ & 0.010 & $3(0$ to 8$)$ & $3(0$ to 9$)$ & 0.199 \\
\hline HASBLED & $2(0$ to 4$)$ & $1(0$ to 4$)$ & $<0.001$ & $1(0$ to 4$)$ & $1(0$ to 4$)$ & 0.089 \\
\hline Recent ischemic stroke/TIA & $4(4.3)$ & $21(7.4)$ & 0.298 & $6(7.9)$ & $15(7.2)$ & 0.846 \\
\hline Recent hemorrhagic stroke & $2(2.2)$ & $0(0)$ & 0.013 & 0 & 0 & \\
\hline Biological prosthesis & $2(2.2)$ & $13(4.6)$ & 0.299 & $9(11.8)$ & $4(1.9)$ & $<0.001$ \\
\hline Metallic prosthesis & $0(0)$ & $7(2.5)$ & 0.126 & $7(9.2)$ & $0(0)$ & $<0.001$ \\
\hline Valvular heart disease & $23(24.7)$ & $70(24.6)$ & 0.987 & $28(36.8)$ & $42(20.2)$ & 0.004 \\
\hline Hemorrhage & $9(9.7)$ & $12(4.2)$ & 0.047 & $5(6.6)$ & $7(3.4)$ & 0.233 \\
\hline Major bleeding & $4(4.3)$ & $5(1.8)$ & 0.164 & $2(2.6)$ & $3(1.4)$ & 0.500 \\
\hline Interaction with DOAC & $60(64.5)$ & $225(79.2)$ & 0.004 & $67(88.2)$ & $158(76)$ & 0.025 \\
\hline Creat. $(\mathrm{mg} / \mathrm{dl}), \mathrm{n}=350$ & $1.1 \pm 0.9$ & $1.1 \pm 0.7$ & 0.986 & $1.3 \pm 1.3$ & $1 \pm 0.3$ & 0.031 \\
\hline LA $(\mathrm{mm}), \mathrm{n}=349$ & $42.6 \pm 7.4$ & $43.5 \pm 6.6$ & 0.283 & $47.3 \pm 6.14$ & $42.2 \pm 6.3$ & $<0.001$ \\
\hline $\operatorname{LVEF}(\%), \mathrm{n}=349$ & $62.1 \pm 12.9$ & $59.8 \pm 15$ & 0.176 & $55.1 \pm 16$ & $61.5 \pm 14.5$ & 0.002 \\
\hline \multicolumn{7}{|l|}{ Arrhythmia reversal } \\
\hline Electric & $10(10.8)$ & $82(28.9)$ & & $19(25)$ & $63(30.3)$ & \\
\hline Not Executed & $41(44.1)$ & $100(35.2)$ & & $40(52.6)$ & $60(28.8)$ & \\
\hline Chemical & $30(32.3)$ & $41(14.4)$ & $<0.001$ & $6(7.9)$ & $35(16.8)$ & 0.005 \\
\hline Spontaneous & $10(10.8)$ & $41(14.4)$ & & $7(9.2)$ & $34(16.3)$ & \\
\hline Ablation & $02(2.2)$ & $20(7)$ & & $4(5.3)$ & $16(7.7)$ & \\
\hline Antiplatelet agents & $56(60.2)$ & $3(15)$ & $<0.001$ & $13(17.1)$ & $30(14.4)$ & 0.577 \\
\hline
\end{tabular}

For continuous variables $n$ ( \pm standard deviation) and for continuous variables $n(\%)$; in case of missing data, total $n$ was placed next to the variable; $p^{*}$ refers to comparison between groups with and without anticoagulants; $p+$ refers to comparison between the groups with VKA and DOAC; TCA: transcutaneous coronary angioplasty; TIA: transient ischemic attack; Creat.: serum creatinine; LA: left atrium; LVEF: left ventricular ejection fraction.

From 2011 to 2015, there was a progressive increase in the use of anticoagulants from $64 \%$ to $87 \%$, largely at the expense of an increase in the use of DOACs, which rose from $29 \%$ to $70 \%$ (a relative increase of $144.8 \%$ ), and reduced use of VKA and antiplatelets alone in the same period. Average annual incorporation of DOACs was $10.4 \%$ (Chart 2). About $25 \%$ of the patients were on antiplatelets alone or combined with anticoagulants.

The prevalence of prescription of oral anticoagulants, antiplatelets and no antithrombotic drugs stratified by risk category according to the $\mathrm{CHA}_{2} \mathrm{DS}_{2}$-VASc and HASBLED scores are shown in charts 3 and 4 . There 


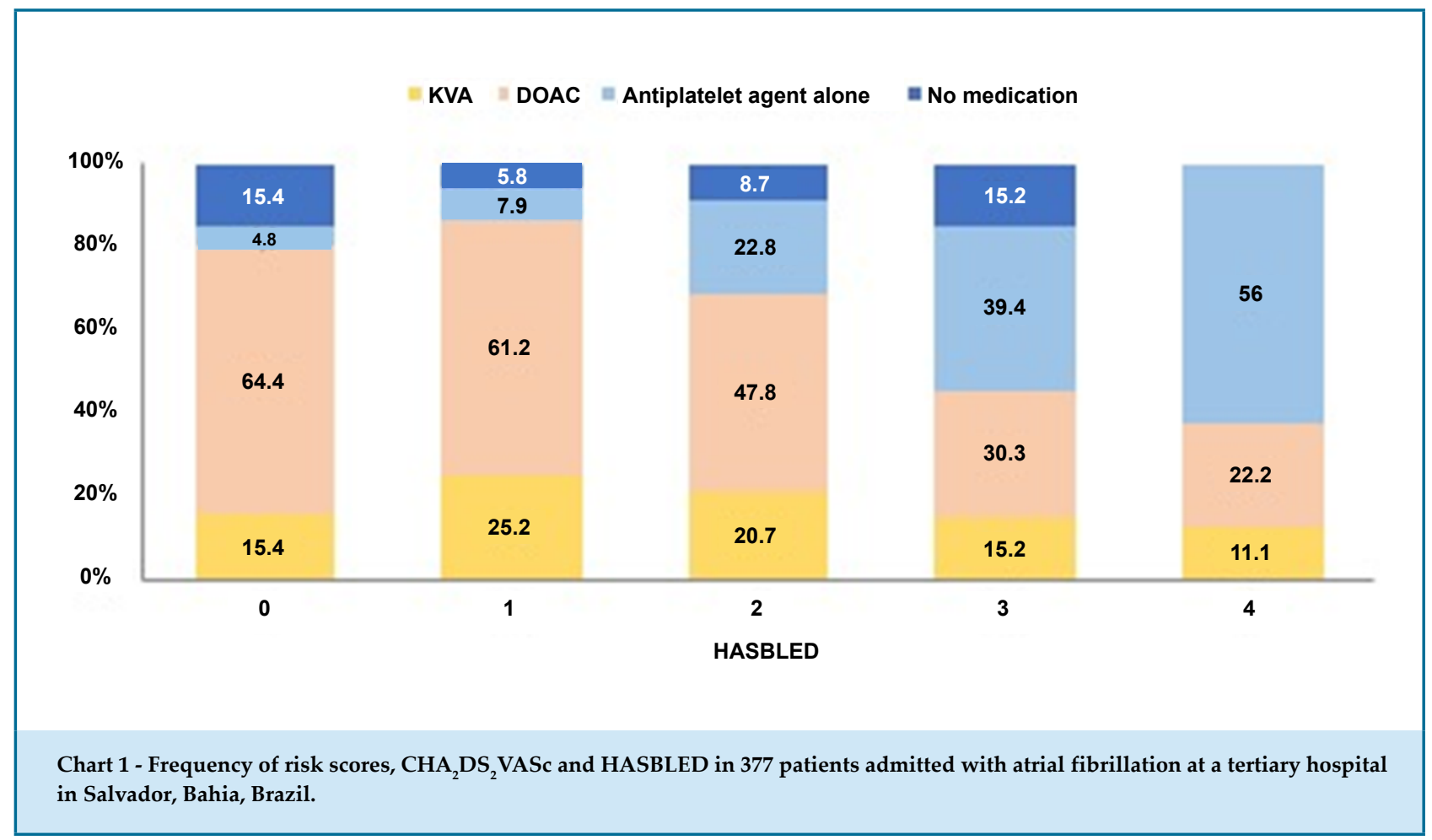

was a strong correlation between the HASBLED and $\mathrm{CHA}_{2} \mathrm{DS}_{2}$-VASc scores (Spearman's r 0.706).

\section{Predictors of use and type of anticoagulants}

Most patients who had anticoagulants prescribed on hospital discharge had a history of previous episodes of AF $(p=0.001)$ and TIA/stroke $(p=0.008)$, were older $(p=0.056)$, had lower mean HASBLED $(p<0.001)$, higher mean weight $(p=0.002)$ and higher prevalence of SAH $(p=0.052)$ than non-anticoagulated patients. In the multivariate logistic regression analysis, the following remained as independent predictors of anticoagulation: previous episodes of AF, SAH and HASBLED $(\mathrm{p}<0.001)$ (Table 2).

To identify variables associated with the prescription of a given class of anticoagulants, we built a multiple logistic regression model using DOAC as a dependent variable and paroxysmal $\mathrm{AF}, \mathrm{CHF}$, serum creatinine, left ventricular ejection fraction, LA diameter, biological valve prosthesis, moderate valvular heart disease and electrical or chemical CV as independent variables. The variables serum creatinine $(\mathrm{p}<0.002)$, LA diameter $(p=0.003)$ and biological valve prosthesis $(p=0.007)$ were inversely associated with the prescription of a DOAC, that is, were predictors of VKA prescription (Table 3).
Appropriateness of the use of anticoagulants

Of the 93 patients with AF whose discharge prescriptions did not contain anticoagulants, 43 had legitimate reasons for doing so: 14 with zero $\mathrm{CHA}_{2} \mathrm{DS}_{2} \mathrm{VASc}, 16$ with absolute contraindications and 13 with relative contraindications. In the other 50 patients (54\%), no reasons were found for the non-prescription of anticoagulants (45 patients) or the reasons on record were inconsistent with the literature ( 5 patients) - "requested by the attending physician"; "already had an indication of using anticoagulants and the attending physician did not prescribe"; "has bronchiectasis and history of bleeding"; "due to the risks of anticoagulation"; "will have pacemaker implanted" (Table 4).

\section{Discussion}

This is the first Brazilian study to demonstrate an increase in the use of oral anticoagulants in patients with $\mathrm{AF}$ as a result of progressive incorporation of DOACs into clinical practice. Despite the high thromboembolic risk of the study population, one-quarter of patients with AF were discharged without anticoagulant prescription, confirming findings from contemporary international registries. However, over the study period 


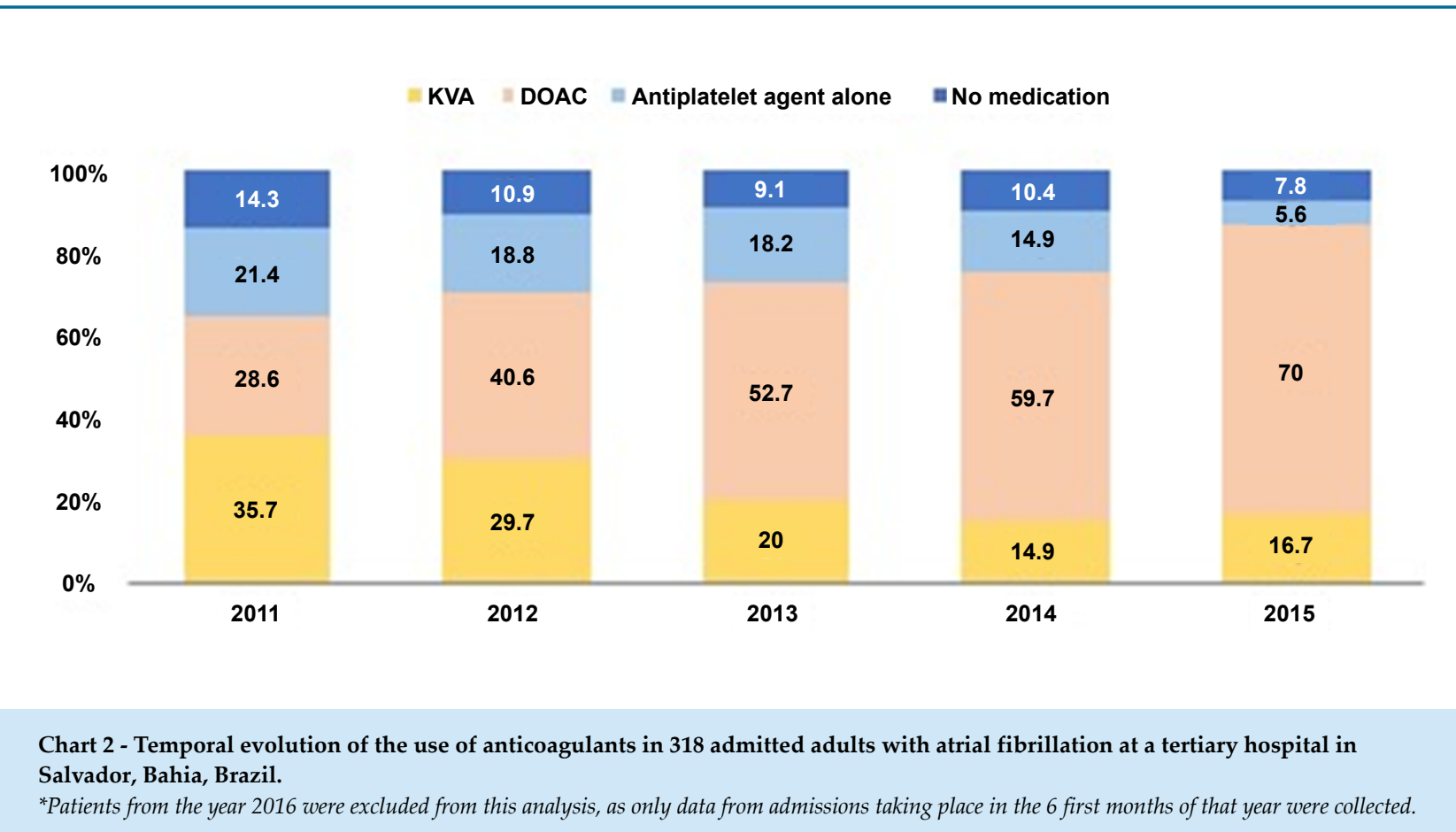

(2011-2016), there was a significant increase in the rate of anticoagulant use, which is largely due to the rapid incorporation of DOACs into clinical practice, which progressively replaced VKAs and antiplatelet agents. We did not find any other Brazilian study showing the evolution of anticoagulation rates in patients with $\mathrm{AF}$ after the introduction of DOACs on the market. A cross-sectional study involving 407 patients with AF treated at the emergence service of a tertiary center in Porto Alegre evaluated the rate of anticoagulant use. Only $34 \%$ of these patients received anticoagulation, and even in patients with $\mathrm{CHA}_{2} \mathrm{DS}_{2}$-VASc $\geq 2$, only $40 \%$ had anticoagulants prescribed. ${ }^{17}$

ANVISA approved the first DOAC in Brazil dabigatran in 2011 - then two new direct inhibitors of factor Xa: rivaroxaban and apixaban. This study documented a rapid incorporation of these new anticoagulants (annual average of $10.4 \%$ ), rising from $28 \%$ in 2011 to $70 \%$ in 2016 . This was the determining factor in the $34.8 \%$ increase in the overall use of anticoagulants in our population of patients with AF.

Most contemporary international registries show the same trend. The GARFIELD AF registry (The Global Anticoagulant Registry in the FIELD-Atrial Fibrillation), which started in 2011, with completed recruitment in follow-up phase, also showed a significant increase in the use of DOACs (4.2\% to $37 \%)$ associated with to an absolute increase of $13.7 \%$ ( $57.4 \%$ to $71.1 \%)$ in the frequency of anticoagulant use. Likewise, the GLORIAAF registry documented a marked increase in the overall rate of anticoagulant use (64\% to $80 \%$ ) between phase 1 and 2 of the study, concomitantly with the introduction and incorporation of DOACs into clinical practice, now used by $48 \%$ of patients, compared to $32 \%$ of VKA users. Both GLORIA AF and GARFIELD AF registries observed a marked decrease in the use of antiplatelet agents. ${ }^{18,19}$

These changes in the pattern of anticoagulant use seem to be occurring in almost all regions of the world, according to GLORIA AF data, except for Asia, where more than $40 \%$ of patients with AF do not receive anticoagulants yet. Also in this registry, Latin America presented high rates of oral anticoagulants and DOACs, of $85 \%$ and $56 \%$, respectively. It seems clear that the greatest factor for the improvement in the global anticoagulation rates in AF observed in international registries and in this study over the past 5 years was the emergence of DOACs. These drugs overcame many difficulties and limitations associated with the practical use of traditional anticoagulants, which strongly impacted the decision on its use.

In addition to the DOACs, the latest therapeutic guidelines for AF may have contributed to an increase in 

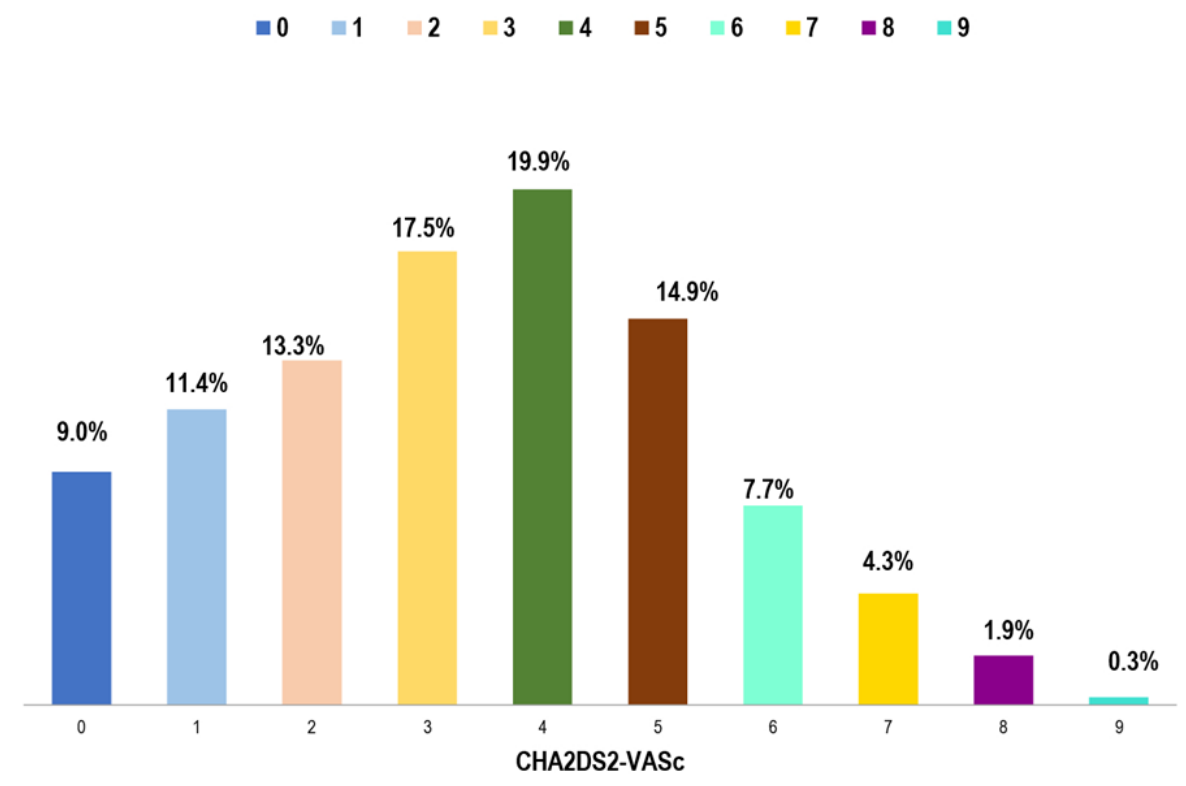

$\because 0=1=2 \backsim 3 \quad \square$

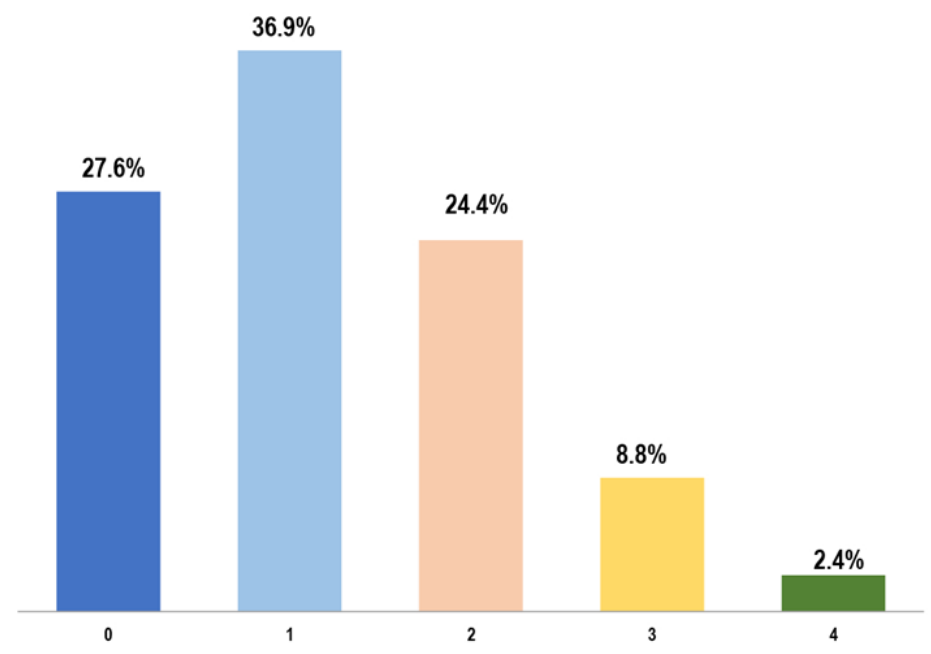

Chart 3 - Frequency of use of anticoagulants regarding the HASBLED score in adult patients admitted with atrial fibrillation at a tertiary hospital in Salvador, Bahia, Brazil.

the number of AF patients receiving oral anticoagulants, since they no longer consider antiplatelet agents as acceptable alternatives to anticoagulants in patients with lower thromboembolic risk. ${ }^{16,20}$

Another important finding of our study was the lack of linearity between thromboembolic risk and the percentage of anticoagulant use. The frequency of anticoagulant prescription increased with the thromboembolic risk score up to $\mathrm{CHA}_{2} \mathrm{DS}_{2}-\mathrm{VASc} 3$. However, in scores $>3$, there was a progressive decrease in the use of anticoagulants. Similar findings were observed in other cohort studies, where anticoagulation rates peaked at $\mathrm{CHA}_{2} \mathrm{DS}_{2}-\mathrm{VASc} 3-4$ scores, followed, unlike our study, with a plateau without an observable decline. ${ }^{18,19}$ Thus, despite the known increase in the annual risk of stroke at each point of the $\mathrm{CHA}_{2} \mathrm{DS}_{2}-\mathrm{VASc}$ 


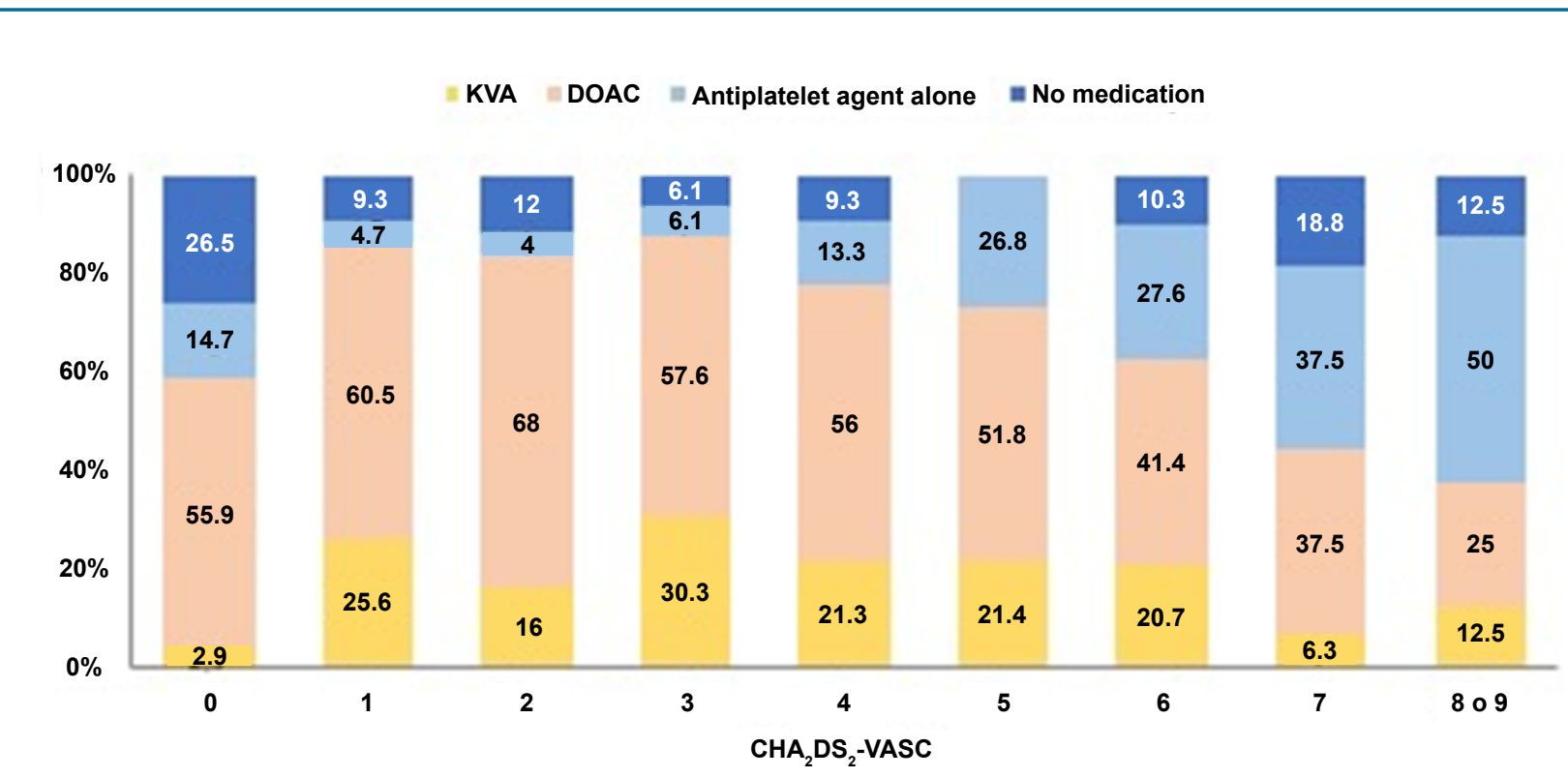

Chart 4 - Frequency of use of anticoagulants regarding the $\mathrm{CHA}_{2} \mathrm{DS}_{2}$-VASC score in adult patients admitted with atrial fibrillation at a tertiary hospital in Salvador, Bahia, Brazil.

Table 2 - Multivariate logistic regression model for predictors of anticoagulant use in patients admitted with atrial fibrillation at a tertiary hospital in Salvador, Bahia, Brazil

\begin{tabular}{lcc}
\hline Variable & OR $(95 \%$ CI $)$ & p \\
\hline History of AF & $3.13(1.80$ to 5.44$)$ & $<0.001$ \\
SAH & $3.05(1.66$ to 5.62$)$ & $<0.001$ \\
HASBLED & $0.50(0.38$ to 0.66$)$ & $<0.001$ \\
\hline
\end{tabular}

Table 3 - Multivariate logistic regression model for predictors of DOAC use in adult patients admitted with atrial fibrillation at a tertiary hospital

\begin{tabular}{lll}
\hline Variable & OR (95\% CI) & $\mathbf{p}$ \\
\hline Serum creatinine (mg/dL) & $0.18(0.06$ to 0.52$)$ & 0.002 \\
LA diameter (mm) & $0.92(0.87$ to 0.97$)$ & 0.003 \\
Biological valve prosthesis & $0.12(0.03$ to 0.57$)$ & 0.007 \\
CV (electrical or chemical) & $2.02(0.97$ to 4.22$)$ & 0.060 \\
\hline
\end{tabular}

Electrical or chemical $C V ; C V$ : electrical cardioversion; LA: left atrium.

Table 4 - Frequency of inappropriate use of DOACs in 208 patients with atrial fibrillation, with indication for use of anticoagulants at a private tertiary hospital in Salvador, Bahia, Brazil

\begin{tabular}{lcccc}
\hline DOAC used & $\begin{array}{c}\text { DOAC misuse } \\
\text { frequency } \\
\mathbf{n}(\%)\end{array}$ & $\begin{array}{c}\text { Contraindication for } \\
\text { drug interaction } \\
\mathbf{n}(\%)\end{array}$ & $\begin{array}{c}\text { Improper dose } \\
\text { reduction } \\
\mathbf{n}(\%)\end{array}$ & $\begin{array}{c}\text { Improper full dose } \\
\mathbf{n}(\%)\end{array}$ \\
\hline Dabigatran $(\mathrm{n}=60)$ & $33(55)$ & $2(3.3)$ & $29(67)$ & $2 / 17(11.8)$ \\
Rivaroxaban $(\mathrm{n}=119)$ & $22(18.5)$ & $1(0.8)$ & $18(48.6)$ & $3 / 82(3.6)$ \\
Apixaban $(\mathrm{n}=29)$ & $8(27.6)$ & $2(6.9)$ & $6(46.1)$ & $0 / 16(0)$ \\
\hline
\end{tabular}


score, $20-50 \%$ of high-risk patients remain unprotected without anticoagulants. Several reasons may have contributed to these findings. Many physicians may not use any tools as structured as $\mathrm{CHA}_{2} \mathrm{DS}_{2}$-VASc for evaluation of thromboembolic risk in AF, using only their imprecise clinical impression ${ }^{12}$ and failing to capture the actual risk of some patients. Besides, patients with higher $\mathrm{CHA}_{2} \mathrm{DS}_{2}$-VASc have a higher prevalence of comorbidities associated with both higher risk of stroke and bleeding risk. Exploring this assumption, we demonstrated a strong correlation between the $\mathrm{CHA}_{2} \mathrm{DS}_{2}-$ VASc and HASBLED scores (Spearman's r 0.706). Physicians may be fearful of prescribing anticoagulants for patients with higher $\mathrm{CHA}_{2} \mathrm{DS}_{2}-\mathrm{VASc}$ if they also present higher risk of bleeding, translated by higher HASBLED. Additionally, since many of these patients are also older and have higher prevalence of atherosclerotic disease, a significant percentage already use antiplatelet agents, which adds to the risk of bleeding and reluctance to prescribe anticoagulants.

\section{Predictors of use of anticoagulants and DOACs}

In our study, history of AF episodes and the presence of SAH individually increased by threefold the chances of patients receiving anticoagulant prescription at discharge. On the other hand, at every 1 point where HASBLED increased, the chance of anticoagulation was reduced by $50 \%$. These are logical and intuitive predictors of anticoagulation, since the first two increase the perception of thromboembolic risk and the last one increases the risk of bleeding. In the NCDR PINNACLE registry, there were more hypertensives among patients taking anticoagulants than among those who did not use anticoagulants $(80 \%$ versus $74 \%$; $\mathrm{p}<0.001) .{ }^{21}$

In an analysis of participants from the 2nd and 5th cohorts of the GARFIELD-AF study in the UK, the two main reasons for the physician to avoid using anticoagulant in patients with $\mathrm{CHA}_{2} \mathrm{DS}_{2}$-VASc $\geq 2$ were risk of fall and hemorrhagic event, respectively. ${ }^{22}$

Regarding the predictors of use of DOACs, we observed that for each $1.0 \mathrm{mg} / \mathrm{dl}$ increase in serum creatinine, there was $82 \%$ less chance of the patient being discharged on DOAC. For 1 year, Luger et al. analyzed AF patients who had stroke or TIA and concluded that the decision on the use of VKA or DOAC was mainly determined by the patient's renal function and absence of previous anticoagulant therapy, both reducing the chance of using DOAC. ${ }^{23}$
In addition to renal function, the presence of a biological valve prosthesis also significantly reduced the chance of DOAC prescription. Although not all studies comparing DOACs with VKA excluded patients with biological prostheses, which do not represent contraindication for the use of DOACs, the unfavorable experience of dabigatran in patients with mechanical prostheses $^{24}$ may cause some fear of using these anticoagulants in the context of any valve prosthesis.

\section{Appropriateness of DOAC prescription}

Our study demonstrated that in $28 \%$ of DOAC prescriptions, the doses were inadequate for the patients' clinical profile. However, as opposed to the findings of the cited studies, the most frequent dosing error was improper reduction of DOAC dose, rather than lack of adjustment. Both the lack of adjustment and improper dose reduction may compromise the efficacy and safety of anticoagulant therapy with DOACs. The randomized cluster study IMPACT-AF showed that multifaceted educational interventions were able to improve the frequency of anticoagulation and reduce the incidence of stroke. ${ }^{25}$

\section{Limitations}

Limitations of this paper include its retrospective design based on electronic medical records, making it subject to registration bias due to lack of information in medical records. In some cases, for example, it was not possible to assess the suitability of the DOAC dose because we did not have serum creatinine, weight or height information. Some patients were excluded because they did not have electronic prescription for discharge. However, these events were infrequent and did not compromise the results found.

Regarding the possibility of generalizing our findings to other populations, although it was conducted in a single center, the characteristics of the study population are similar to those of major international registries of AF. However, because it was conducted in a private medical center, our data cannot be extrapolated to patients with AF from the public healthcare system.

\section{Conclusions}

This study demonstrated that, following the approval of DOACs for clinical use in Brazil in 2011, these anticoagulants were rapidly incorporated into clinical 
practice, becoming the therapy of choice for patients with AF and contributing decisively to the increase of anticoagulation rates in this group of patients. Despite this important achievement, $13 \%$ of eligible patients remained without anticoagulation. Additionally, besides the long-standing historical difficulty of keeping patients on VKA in the narrow therapeutic range, at least $25 \%$ of patients using DOACs are exposed to inappropriate doses, compromising its efficacy and safety.

The data and reflections described in this study should serve to guide the leaders of our healthcare system and medical societies towards building educational and awareness-raising strategies for health professionals and patients regarding the importance of correct prescription and adherence to anticoagulant therapy in AF.

\section{Author contributions}

Conception and design of the research: Geraldes MFA, Darze ES, Rocha PN. Acquisition of data: Geraldes MFA. Analysis and interpretation of the data: Geraldes MFA, Darze ES, Rocha PN. Statistical analysis: Geraldes MFA, Darze ES, Rocha PN. Writing of the manuscript: Geraldes MFA, Darze ES, Rocha PN. Critical revision of the manuscript for intellectual content: Geraldes MFA, Darze ES, Rocha PN.

\section{Potential Conflict of Interest}

No potential conflict of interest relevant to this article was reported.

\section{Sources of Funding}

There were no external funding sources for this study.

\section{Study Association}

This article is part of the thesis of master submitted by Maria de Fátima de Araújo Geraldes, from Universidade Federal da Bahia.

\section{Ethics approval and consent to participate}

This study was approved by the Ethics Committee of the Prof. Celso Figuerôa, Hospital Santa Izabel under the protocol number 917.116. All the procedures in this study were in accordance with the 1975 Helsinki Declaration, updated in 2013. Informed consent was obtained from all participants included in the study.

\section{References}

1. Benjamin EJ, Wolf PA, Agostino RBD, Silbershatz H, Kannel WB, Levy D. Clinical Investigation and Reports Impact of Atrial Fibrillation on the Risk of Death The Framingham Heart Study. Circulation. 1998;98(10):946-53.

2. Krahn AD, Manfreda J, Tate RB, Mathewson FAL, Cuddy TE. The natural history of atrial fibrillation: Incidence, risk factors, and prognosis in the manitoba follow-up study. Am J Med.1995;98(5):476-84.

3. Psaty BM, Manolio TA, Kuller LH, Kronmal RA, Cushman M, Fried LP, et al. Incidence of and Risk Factors for Atrial Fibrillation in Older Adults. Circulation. 1997;96(7):2455-61.

4. Vidaillet H, Granada JF, Chyou P o-H, Maassen K, Ortiz M, Pulido JN, et al. A population-based study of mortality among patients with atrial fibrillation or flutter. Am J Med [Internet]. 2002;113(5):365-70.

5. Stewart S, Hart CL, Hole DJ, McMurray JJ. A population-based study of the long-term risks associated with atrial fibrillation: 20-year follow-up of the Renfrew/Paisley study. Am J Med. 2002;113(5):359-64.

6. Go AS, Hylek EM, Phillips KA, Chang Y, Henault LE, Selby J V., et al. Prevalence of Diagnosed Atrial Fibrillation in Adults. JAMA. 2001;285(18):2370-75.

7. Hart RG, Pearce LA, Aguilar MI. Meta-analysis: antithrombotic therapy to prevent stroke in patients who have nonvalvular atrial fibrillation. Ann Intern Med. 2007;146(12):857-67.

8. Fornari LS, Calderaro D, Nassar IB, Lauretti C, Nakamura L, Bagnatori $\mathrm{R}$, et al. Misuse of antithrombotic therapy in atrial fibrillation patients: Frequent, pervasive and persistent. J Thromb Thrombolysis. 2007 Feb;8:65-71.

9. Dewilde S, Carey IM, Emmas C, Richards N. Trends in the prevalence of diagnosed atrial fibrillation, its treatment with anticoagulation and predictors of such treatment in UK primary care. Heart. 2006;92(8):1064-70.

10. Wan Y, Heneghan C, Perera R, Roberts N, Hollowell J, Glasziou P, et al Anticoagulation control and prediction of adverse events in patients with atrial fibrillation: A systematic review. Circ Cardiovasc Qual Outcomes. 2008;1(2):84-91.

11. Patel MR, Mahaffey KW, Garg J, Pan G, Singer DE, Hacke W, et al. Rivaroxaban versus Warfarin in Nonvalvular Atrial Fibrillation. n engl j medJLH); R Perth Hospi-tal N Engl J Med 2011;36510365(10):883-91.

12. Lip GYH, Nieuwlaat R, Pisters R, Lane DA, Crijns HJGM. Refining clinical risk stratification for predicting stroke and thromboembolism in atrial fibrillation using a novel risk factor-based approach: The Euro Heart Survey on atrial fibrillation. Chest. 2010;137(2):263-72.

13. Lip GYH, Frison L, Halperin JL, Lane DA. Comparative validation of a novel risk score for predicting bleeding risk in anticoagulated patients with atrial fibrillation: The HAS-BLED (hypertension, abnormal renal/ liver function, stroke, bleeding history or predisposition, labile INR, elderly, drug. J Am Coll Cardiol. 2011;57(2):173-80.

14. Yao X, Gersh BJ, Sangaralingham LR, Kent DM, Shah ND, Abraham NS, et al. Comparison of the CHA2DS2-VASc, CHADS2, HAS-BLED, ORBIT, and ATRIA Risk Scores in Predicting Non-Vitamin K Antagonist Oral Anticoagulants-Associated Bleeding in Patients With Atrial Fibrillation. Am J Cardiol 2017;120(9):1549-56. 
15. Magalhães LP, Figueiredo MJO, Cintra FD, Saad EB, Kuniyoshi RR, Teixeira RA, et al. II Diretrizes Brasileiras de Fibrilação Atrial. Arq Bras Cardiol. 2016;106(4):1-22.

16. Steffel J, Verhamme P, Potpara T, Albaladejo P, Antz M, Desteghe et al. ESC Scientific Document Group; The 2018 European Heart Rhythm Association Practical Guide on the use of non-vitamin K antagonist oral anticoagulants in patients with atrial fibrillation. European Heart Journal.2018; 39(16):1330-1393.

17. Almeida ED, Guimarães RB, Stephan LS, Medeiros AK, Foltz K, Santanna RT, et al. Clinical Differences between Subtypes of Atrial Fibrillation and Flutter: Cross-Sectional Registry of 407 Patients. Arq Bras Cardiol. 2015;105(1) 3-10.

18. Huisman MV, Rothman KJ, Paquette M, Teutsch C, Diener HC, Dubner SJ, et al. The Changing Landscape for Stroke? Prevention in AF: Findings From the GLORIA-AF Registry Phase 2. J Am Coll Cardiol. 2017;69(7):777-85.

19. Ten Cate $\mathrm{V}$, ten Cate $\mathrm{H}$, Verheugt FWA. The global anticoagulant registry in the FIELD-atrial fibrillation (GARFIELD-AF): Exploring the changes in anticoagulant practice in patients with non-valvular atrial fibrillation in the Netherlands. Netherlands Hear J. 2016;24(10):574-80.

20. Nishimura RA, Otto CM, Bonow RO, Carabello BA, Erwin JP, Fleisher LA, et al. 2017 AHA/ACC Focused Update of the 2014 AHA/ACC Guideline for the Management of Patients With Valvular Heart Disease. J Am Coll Cardiol. 2017; 70(2):252-289.
21. Hsu JC, Maddox TM, Kennedy KF, Katz DF, Marzec LN, Lubitz SA, Gehi AK, Turakhia MP, Marcus GM. Oral Anticoagulant Therapy Prescription in Patients With Atrial Fibrillation Across the Spectrum of Stroke RiskInsights From the NCDR PINNACLE Registry. JAMA Cardiol. 2016;1(1):55-62.

22. Apenteng PN, Gao H, Hobbs FR, Fitzmaurice DA, UK GARFIELD-AF Investigators and GARFIELD-AF Steering Committee. Temporal trends in antithrombotic treatment of real-world UK patients with newly diagnosed atrial fibrillation: findings from the GARFIELD-AF registry. BMJ Open 2018;8(1):e 018905.

23. Luger S, Hohmann C, Kraft P, Halmer R, Gunreben I, Neumann-Haefelin T, et al. Prescription Frequency and Predictors for the Use of Novel Direct Oral Anticoagulants for Secondary Stroke Prevention in the First Year after Their Marketing in Europe - A Multicentric Evaluation. Int J Stroke. 2014;9(5):569-75.

24. Eikelboom JW, Connolly SJ, Brueckmann M, Granger CB, Kappetein AP, Mack MJ, et al. Dabigatran versus Warfarin in Patients with Mechanical Heart Valves. N Engl J Med. 2013;369(13):1206-14.

25. Rao MP, Ciobanu AO, Lopes RD, Fox KA, Xian Y, Pokorney SD, et al. A clustered randomized trial to IMProve treatment with AntiCoagulanTs in patients with Atrial Fibrillation (IMPACT-AF): Design and rationale. Am Heart J. 2016 Jun;176:107-13. 